\title{
A AIDS nas regiões de fronteira no Brasil de 1990 a 2003
}

\author{
Antonio Luiz Rodrigues-Júnior ${ }^{1}$ e Euclides Ayres de Castilho ${ }^{2}$
}

Como citar Rodrigues-Júnior AL, Castilho EA. A AIDS nas regiões de fronteira no Brasil de 1990 a 2003. Rev Panam Salud Publica. 2009;25(1):31-8.

RESUMO Objetivo. Estudar a epidemia de AIDS na faixa de fronteira no Brasil, com enfoque espacial e temporal.

Métodos. Trata-se de um estudo ecológico no qual os casos de AIDS notificados ao Ministério da Saúde de 1990 a 2003 foram agrupados conforme as áreas de fome estabelecidas por Josué de Castro nos anos 1940 e conforme 19 sub-regiões culturais. A avaliação espacial foi feita com base nos coeficientes de incidência para os municípios da faixa de fronteira; a avaliação temporal considerou o número absoluto de casos em periodos trimestrais de 1990 a 2003 em cada área de fome considerada (Extremo Sul, Centro-Oeste e Amazônica).

Resultados. Foram notificados 7973 casos de AIDS na faixa de fronteira brasileira: 648 na área Amazônica, 1579 no Centro-Oeste e 5746 no Extremo Sul (população de 668 098, 895489 e 2769361 habitantes, respectivamente). As sub-regiões com os maiores coeficientes de incidência de AIDS, em cada área, estavam localizadas nas fronteiras tríplices entre o Brasil e outros países da América do Sul. Houve predominância da transmissão por via sexual, sendo a transmissão heterossexual a categoria mais freqüente, seguida pela de transmissão por homens que fazem sexo com outros homens. Essas duas categorias representaram $87,2 \%$ dos casos notificados. Quanto à análise temporal, as estimativas do parâmetro de tendência foram $0,53$ ( $\mathrm{P}<0,0001), 0,83(\mathrm{P}<0,0001)$ e 3,47 ( $\mathrm{P}<0,0001)$, respectivamente, para a área Amazônica, Centro-Oeste e Extremo Sul.

Conclusão. O aperfeiçoamento dos serviços de saúde na faixa de fronteira brasileira, em toda a sua extensão, pode ser uma estratégia de integração territorial e de enfrentamento da epidemia de AIDS, desde que as diferenças sociais, econômicas e culturais sejam consideradas.

Palavras-chave HIV, síndrome de imunodeficiência adquirida, áreas de fronteira, estudos ecológicos, Brasil.

Em 2005, com o propósito de integrar as ações e os serviços de saúde nas regiões de fronteira, o Ministério da Saúde

\footnotetext{
1 Universidade de São Paulo, Medicina Social. Correspondência: Avenida Bandeirantes, CEP 14049900, Ribeirão Preto, SP, Brasil. Fone: +55-163602.2537; fax: +55-16-3633.1586 3602.3070; e-mail: alrj@fmrp.usp.br

2 Universidade de São Paulo, Faculdade de Medicina, Departamento de Medicina Preventiva.
}

brasileiro instituiu o Sistema Integrado de Saúde das Fronteiras (SIS Fronteiras) (1). A noção de fronteira não se baseia somente em compartimentos geopolíticos, mas, também, diz respeito a um espaço geográfico de interação cultural, onde ocorrem fluxos migratórios, práticas legais e ilegais, guerrilhas e degradação do espaço geográfico (2-4). Em 1979, foi definida a faixa de fronteira brasileira (5), criando uma área de grande extensão territorial caracterizada por diferenças sociais, econômicas e culturais.

Peiter (6) estudou a faixa de fronteira terrestre brasileira a partir da perspectiva de Josué de Castro (7), que, na década de 1940, definiu cinco áreas de fome no Brasil: o Nordeste Açucareiro, o Sertão do Nordeste, a área Amazônica, o CentroOeste e o Extremo Sul, sendo que as três últimas incluem fronteiras terrestres. Dessa forma, Peiter identificou epicen- 
tros da epidemia de AIDS e as chamadas "cidades-gêmeas", onde ocorrem interações sociais entre populações vizinhas, considerando 19 regiões culturais.

Na década de 1990, a epidemia de AIDS no Brasil motivou o interesse dos epidemiologistas pelo padrão de difusão espacial da doença, caracterizado pelos fenômenos de "interiorização", "feminização", "pauperização" e "juvenilização" (8). Figuram, entre os fatores determinantes da difusão da epidemia, os movimentos migratórios motivados pela busca de melhores condições de vida ou de oportunidades de trabalho ou pela necessidade de evadir áreas de conflitos (2); o narcotráfico, que, pelo barateamento da droga, gera usuários no trajeto de escoamento, tornando-a acessível aos indivíduos vulneráveis (9); e o comércio do sexo, que, no contexto de vulnerabilidade, expõe os indivíduos à prática do sexo não seguro, entre outros (10). Barcellos et al. (11), em estudo sobre a epidemia de AIDS na fronteira brasileira, encontraram altas incidências nos municípios de Uruguaiana, Estado do Rio Grande do Sul (68,7 por $10^{5}$ habitantes), Foz do Iguaçu, Estado do Paraná (41,4 por $10^{5}$ habitantes), Corumbá, Estado do Mato Grosso do Sul (33,4 por $10^{5}$ habitantes), Oiapoque, Estado do Amapá (15,5 por $10^{5}$ habitantes) e Tabatinga, Estado do Amazonas (10,6 por $10^{5}$ habitantes). Entretanto, além do enfoque da distribuição espacial, a tendência da epidemia da AIDS pode ser estudada desde um ponto de vista temporal, com o uso de modelos de regressão autocorrelacionados (12).

O objetivo do presente trabalho foi realizar uma avaliação espacial e temporal da epidemia de AIDS nos municípios da faixa de fronteira brasileira pelo agrupamento dos casos notificados ao Ministério da Saúde entre 1990 e 2003 segundo as áreas de fome e as sub-regiões culturais propostas anteriormente.

\section{MATERIAIS E MÉTODOS}

Foi realizado um estudo ecológico de base territorial considerando apenas os municípios situados na faixa de fronteira do Brasil (5). Foi considerada a descrição atualizada feita pelo Programa de Desenvolvimento da Faixa de Fronteira (13). No presente estudo, admitiu-se que as "áreas de fome" são representativas de diferenças regionais históricas em nosso país, de forma que os municípios da faixa de fronteira foram reagrupados segundo essas áreas. Conforme descrito por Castro (7), a área Amazônica foi definida como sendo de fome endêmica, com um padrão alimentar deficiente, baseado no consumo de peixes e de mandioca, que não provê a ingestão das necessidades mínimas de nutrientes. A área CentroOeste foi caracterizada por um padrão alimentar incompleto, apesar de muito calórico, baseado no consumo de proteína animal, gorduras e milho e deficiente em vitaminas e fibras. O padrão alimentar do Extremo Sul foi caracterizado pelo sobrepeso e pela obesidade.

Em seguida, as áreas de fome foram subdivididas em sub-regiões culturais, constituindo 19 regiões homogêneas em escala local, seguindo a descrição de Peiter (6) e do Programa de Desenvolvimento da Faixa de Fronteira (13). Assim, a área Amazônica foi dividida nas sub-regiões Oiapoque-Tumucumaque, Campos do Rio Branco, Parima-Alto Rio Negro, Alto Solimões, Alto Juruá e Vale do Acre-Purus; a área Centro-Oeste foi dividida nas sub-regiões MadeiraMamoré, Fronteira do Guaporé, Chapada dos Parecis, Alto Paraguai, Pantanal, Bodoquena, Dourados e Cone Sul Matogrossense; e o Extremo Sul foi dividido nas sub-regiões Portal do Paraná, Sudoeste do Paraná, Oeste de Santa Catarina, Noroeste do Rio Grande do Sul e Fronteira Metade Sul do Rio Grande do Sul.

As informações sobre casos de AIDS foram obtidas no banco de dados do Sistema Nacional de Agravos de Notificação (SINAN) do Ministério da Saúde pelo Programa Nacional de Doenças Sexualmente Transmissíveis e AIDS (PN-DST/ AIDS). Esse banco de dados contém os registros dos casos de AIDS notificados entre $1^{\circ}$ de janeiro de 1990 e 31 de dezembro de 2003, exceto a identificação do indivíduo, garantindo a confidencialidade das informações secundárias (14). Foram consideradas, também, as estimativas de adultos e crianças vivendo com HIV/ AIDS em países da América do Sul feitas pelo Programa Conjunto das Nações Unidas sobre HIV/AIDS (UNAIDS) em 2003 e divulgadas através de planilha eletrônica pelo site http://www.unaids.org/. Essas estimativas foram usadas com a finalidade de caracterizar a paisagem epidemiológica da AIDS na qual estão inseridos os municípios da faixa de fronteira brasileira.

Adotando as estimativas de tamanhos populacionais municipais do Departa- mento de Informática do Sistema Único de Saúde do Ministério da Saúde (DATASUS), foram calculados os coeficientes de incidência de AIDS de 1990 a 2003 para elaborar os mapas usados na avaliação espacial da epidemia (15), considerando os municípios e as sub-regiões culturais como unidades ecológicas. Os coeficientes são medidas apropriadas às técnicas de visualização através de mapas de áreas ou de polígonos irregulares (16).

O número absoluto de casos de AIDS foi subdividido em 52 períodos trimestrais, de $1^{\circ}$ de janeiro de 1990 a 31 de dezembro de 2003, formando séries temporais $Y(t)$, que foram usadas para estudar a evolução temporal da epidemia em cada área de fome da faixa de fronteira brasileira. Optamos por usar os valores absolutos para preservar a magnitude da epidemia de AIDS em cada área de fome. As séries trimestrais de cada área foram analisadas através de um modelo linear autorregressivo de primeira ordem, com o objetivo de verificar se, ao longo do período estudado, a incidência tendeu ao aumento, à diminuição ou à estabilização, pela seguinte equação:

$$
Y(t)=\beta_{0}+\beta_{1} t+\rho \varepsilon(t-1)
$$

onde $Y(t)$ representa o número absoluto, no trimestre $t$, de casos que formam uma coleção de variáveis aleatórias indexadas pelo tempo $\{t=1,2,3, \ldots, 52\}$; $\rho$ representa o coeficiente de autocorrelação, que define a dependência existente entre incidências trimestrais consecutivas, $Y(t)$ e $Y(t+1)$, ou entre os respectivos erros do modelo, $\varepsilon(t)$ e $\varepsilon(t+1)$; o modelo é chamado de primeira ordem quando a autocorrelação se dá pelo período imediatamente anterior à observação (11). $\mathrm{O}$ programa STATA ${ }^{\circledR}$ foi usado para realizar os cálculos estatísticos.

\section{RESULTADOS}

Foram notificados 7973 casos de AIDS na faixa de fronteira brasileira, sendo 648 na área Amazônica, 1579 no CentroOeste e 5746 no Extremo Sul, considerando os totais populacionais de 668 098, 895489 e 2769361 habitantes, respectivamente. Destacaram-se, pela magnitude da incidência acumulada (tabela 1): na área Amazônica, as sub-regiões de Campos do Rio Branco, com 278 casos, na tríplice fronteira Venezuela-GuianaBrasil, e do Vale do Acre-Alto Purus, com 212 casos, na tríplice fronteira Peru- 
TABELA 1. Distribuição dos casos de AIDS na faixa de fronteira brasileira segundo sub-regiões e categorias de exposição, Brasil, 1990 a 2003

\begin{tabular}{|c|c|c|c|c|c|c|c|c|c|c|}
\hline Sub-região & População & AIDS $^{a}$ & $\begin{array}{l}\text { Sexo com } \\
\text { homens }^{b}\end{array}$ & Bissexual & $\begin{array}{l}\text { Heteros- } \\
\text { sexual }\end{array}$ & Drogas $^{c}$ & Hemofilia & Transfusão & Perinatal & Ignorado \\
\hline \multicolumn{11}{|l|}{ Área Amazônica } \\
\hline Oiapoque-Tumucumaque & 16481 & 92 & 5 & 8 & 59 & 9 & 1 & 2 & 1 & 7 \\
\hline Campos do Rio Branco & 81505 & 278 & 29 & 31 & 155 & 18 & $\ldots$ & 2 & 10 & 33 \\
\hline Parima-Alto Rio Negro & 89017 & 9 & $\ldots$ & 1 & 6 & $\ldots$ & $\ldots$ & 1 & 1 & $\ldots$ \\
\hline Alto Solimões & 140807 & 49 & 4 & 9 & 33 & $\ldots$ & $\ldots$ & $\ldots$ & $\ldots$ & 3 \\
\hline Alto Juruá & 119263 & 8 & 1 & $\ldots$ & 5 & 1 & $\ldots$ & $\ldots$ & 1 & $\ldots$ \\
\hline Vale do Acre-Alto Purus & 221025 & 212 & 46 & 14 & 130 & 6 & $\ldots$ & 1 & 6 & 8 \\
\hline \multicolumn{11}{|l|}{ Centro-Oeste } \\
\hline Madeira-Mamoré & 32274 & 535 & 36 & 43 & 341 & 34 & 1 & 13 & 12 & 55 \\
\hline Fronteira Guaporé & 139444 & 36 & 1 & 3 & 24 & 3 & $\ldots$ & $\ldots$ & 2 & 3 \\
\hline Chapada dos Parecis & 70362 & 153 & 3 & 10 & 101 & 26 & $\ldots$ & 2 & 4 & 7 \\
\hline Alto Paraguai & 120096 & 53 & 1 & 5 & 41 & 3 & $\ldots$ & $\ldots$ & $\ldots$ & 3 \\
\hline Pantanal & 121742 & 316 & 28 & 15 & 163 & 57 & 2 & 2 & 20 & 29 \\
\hline Bodoquena & 92804 & 42 & 3 & 1 & 28 & 1 & $\ldots$ & $\ldots$ & 5 & 4 \\
\hline Dourados & 203926 & 291 & 31 & 12 & 166 & 41 & $\ldots$ & 1 & 11 & 29 \\
\hline Cone Sul-Matogrossense & 114841 & 153 & 5 & 4 & 95 & 19 & 1 & 3 & 10 & 16 \\
\hline \multicolumn{11}{|l|}{ Extremo Sul } \\
\hline $\begin{array}{l}\text { Portal do Paraná } \\
\text { Vale Sulinos-Sudoeste }\end{array}$ & 234563 & 173 & 12 & 7 & 108 & 22 & 1 & 2 & 13 & 8 \\
\hline $\begin{array}{l}\text { Paraná } \\
\text { Vales Sulinos-Oeste S. }\end{array}$ & 991946 & 1684 & 87 & 78 & 1150 & 271 & $\ldots$ & 4 & 67 & 27 \\
\hline $\begin{array}{l}\text { Catarina } \\
\text { Vales Sulinos-Noroeste }\end{array}$ & 496218 & 481 & 24 & 28 & 332 & 49 & 1 & 1 & 30 & 16 \\
\hline Rio Grande do Sul & 847585 & 731 & 52 & 23 & 398 & 120 & 2 & 5 & 41 & 90 \\
\hline Fronteira Metade Sul & 199049 & 2677 & 265 & 162 & 1270 & 579 & 9 & 6 & 134 & 252 \\
\hline
\end{tabular}

a Número acumulado de casos de AIDS

${ }^{b}$ Homens que fazem sexo com outros homens.

c Usuários de drogas injetáveis.

Bolívia-Brasil; na área Centro-Oeste, as sub-regiões Madeira-Mamoré, com 535 casos, muito próxima à tríplice fronteira Peru-Bolívia-Brasil, e Pantanal, com 316 casos, na tríplice fronteira Bolívia-Paraguai-Brasil; e no Extremo Sul, as sub-regiões Vales Sulinos-Sudoeste do Paraná, com 1684 casos, na tríplice fronteira Paraguai-Argentina-Brasil, e Fronteira Metade Sul do Rio Grande do Sul, na tríplice fronteira Uruguai-ArgentinaBrasil, com 2677 casos. A figura 1 apresenta o mapa temático da distribuição da incidência acumulada nas 19 sub-regiões culturais no ano de 2003.

Como se pode deduzir da tabela 1, na área Amazônica 5,3\% dos casos usavam drogas injetáveis, contra 11,7\% dos casos na área Centro-Oeste e 18,1\% dos casos na área Extremo Sul. Merecem destaque as incidências de AIDS, nesta categoria de transmissão, nas subregiões Fronteira Metade Sul do Rio Grande do Sul, com 579 casos, e Vales Sulinos do Sudoeste do Paraná, com 271 casos. A categoria de transmissão heterossexual foi observada em 57,8\% dos casos e representa a categoria de exposição mais freqüente, apontando para o caráter da "heterossexualização".

O padrão epidemiológico da incidência de AIDS nos municípios da faixa de fronteira no Brasil mostrou a predominância da transmissão por via sexual, sendo a categoria de transmissão heterossexual a mais freqüente, seguida pela categoria de transmissão de homens que fazem sexo com outros homens (HSH); dos 7973 casos notificados, $6952(87,2 \%)$ foram relacionados a essas categorias. A análise descritiva da epidemia de AIDS segundo as categorias de transmissão mostrou a feminização e a heterossexualização nas três áreas, com razão de sexo de 1,14 na área Amazônica, de 1,17 na área Centro-Oeste e de 1,34 na área Extremo Sul. Houve, na área Amazônica, o predomínio de casos nas categorias de exposição sexual nas sub-regiões que apresentam divisa com a Bolívia, o Peru, a Colômbia e a Venezuela, excetuando-se a sub-região Oiapoque-Tumucumaque, que faz fronteira com a Guiana, o Suriname e a Guiana Francesa.

$\mathrm{Na}$ categoria de pessoas que usavam drogas injetáveis, houve um maior número de casos masculinos, mostrando uma razão de sexo de 3,86 homens para cada mulher na área Amazônica, 3,48 na área Centro-Oeste e 4,45 na área Extremo Sul. Destaca-se a maior freqüência de homens nessa categoria de transmissão em municípios da área Extremo Sul cujo território faz divisas com Paraguai, Argen- tina e Uruguai. Na área Centro-Oeste, as pessoas que usavam drogas injetáveis também vieram logo depois dos heterossexuais, mas as sub-regiões Fronteira Guaporé, Alto Paraguai e Bodoquena apresentaram poucos casos, talvez devido à subnotificação pelos municípios que têm divisas com o Paraguai e a Bolívia.

Os modelos autorregressivos, que foram ajustados às séries trimestrais de incidência de AIDS nas três áreas, forneceram as estimativas dos parâmetros do modelo matemático, em especial das tendências das séries. A figura 2 apresenta os modelos (linhas) ajustados aos dados observados (pontos): as estimativas do parâmetro de tendência foram $0,53(P<0,0001), 0,83(P<0,0001)$ e 3,47 $(P<0,0001)$, respectivamente, para a área Amazônica, Centro-Oeste e Extremo Sul. Essas estimativas indicam a velocidade com que a epidemia se disseminou, ou seja, indicam o incremento médio na incidência acumulada trimestral, em cada área, no período de estudo. A figura 2 também ilustra a adequação do modelo temporal de primeira ordem para modelar as séries trimestrais das áreas de fome. As figuras 3, 4 e 5 mostram a distribuição da incidência acumulada de AIDS nos municípios pertencentes à faixa de fronteira brasileira em cada área. 
FIGURA 1. Distribuição espacial do coeficiente de incidência de AIDS nas sub-regiões brasileiras que apresentam divisas com países da América do Sul e estimativas de pessoas vivendo com HIV/AIDS nesses países, 2003
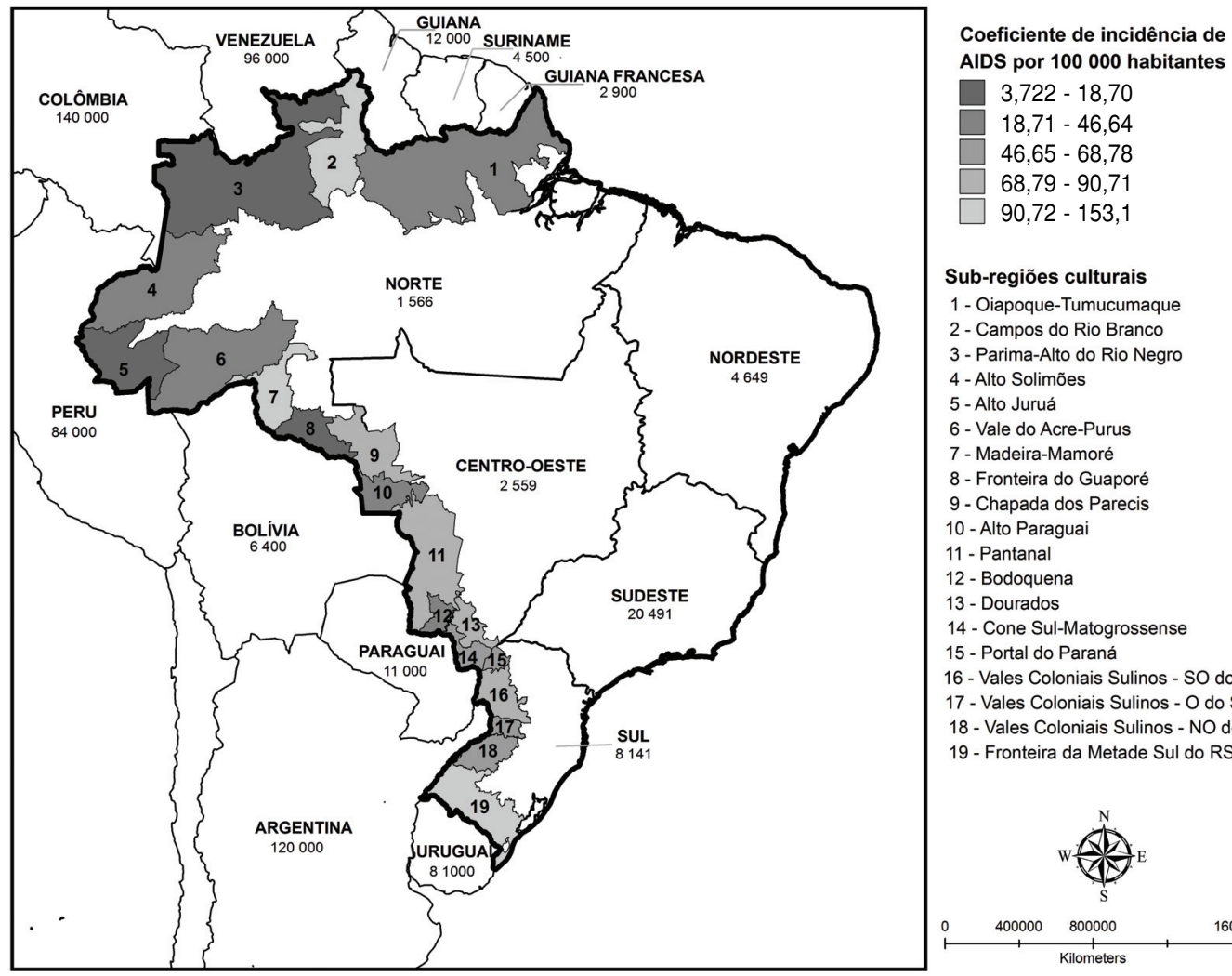

Sub-regiões culturais

1 - Oiapoque-Tumucumaque

2 - Campos do Rio Branco

3 - Parima-Alto do Rio Negro

4 - Alto Solimões

5 - Alto Juruá

6 - Vale do Acre-Purus

7 - Madeira-Mamoré

8 - Fronteira do Guaporé

9 - Chapada dos Parecis

10 - Alto Paraguai

11 - Pantanal

12 - Bodoquena

13 - Dourados

14 - Cone Sul-Matogrossense

15 - Portal do Paraná

16 - Vales Coloniais Sulinos - SO do PR

17 - Vales Coloniais Sulinos - O do SC

18 - Vales Coloniais Sulinos - NO do RS

19 - Fronteira da Metade Sul do RS

Fonte: Dados de incidência de AIDS na América Latina obtidos do Joint United Nations Programme on HIV/AIDS (UNAIDS), 2003.

FIGURA 2. Diagrama de dispersão do ajuste dos modelos autorregressivos da incidência de AIDS em áreas de fronteira, Brasil, 1990 a 2003
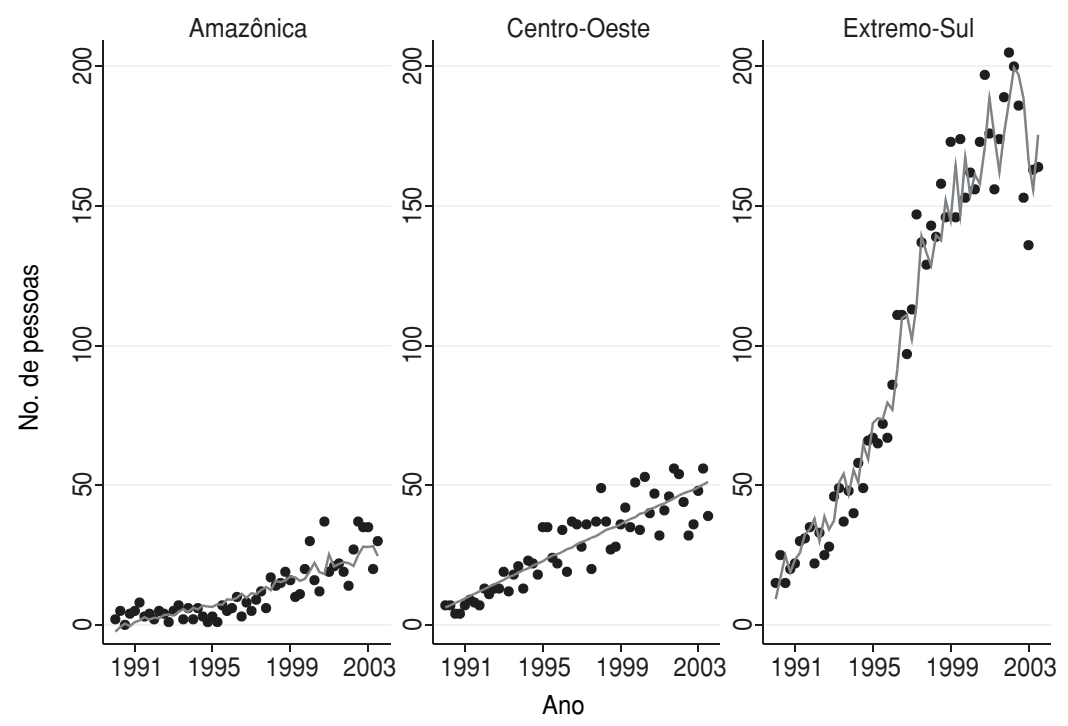

\section{DISCUSSÃO}

A migração humana é um tema complexo, especialmente nas faixas de fronteira, nas áreas de fome e nas regiões de tríplices fronteiras, onde a porosidade geopolítica é maior e onde a mobilidade transfronteiriça é mais comum; desnecessário dizer que abordar tal complexidade implica, inevitavelmente, uma limitação deste estudo. Na Região Norte, a tríplice fronteira Venezuela-Guiana-
Brasil (Estado de Roraima) está situada numa região caracterizada pela ocupação de "espaços vazios" pelos madeireiros, pelos garimpeiros e pelos fazendeiros, apresentando um maior fluxo de brasileiros em direção à Venezuela e à 
FIGURA 3. Distribuição espacial do coeficiente de incidência de AIDS na faixa de fronteira da área Amazônica, Brasil, 1990 a 2003

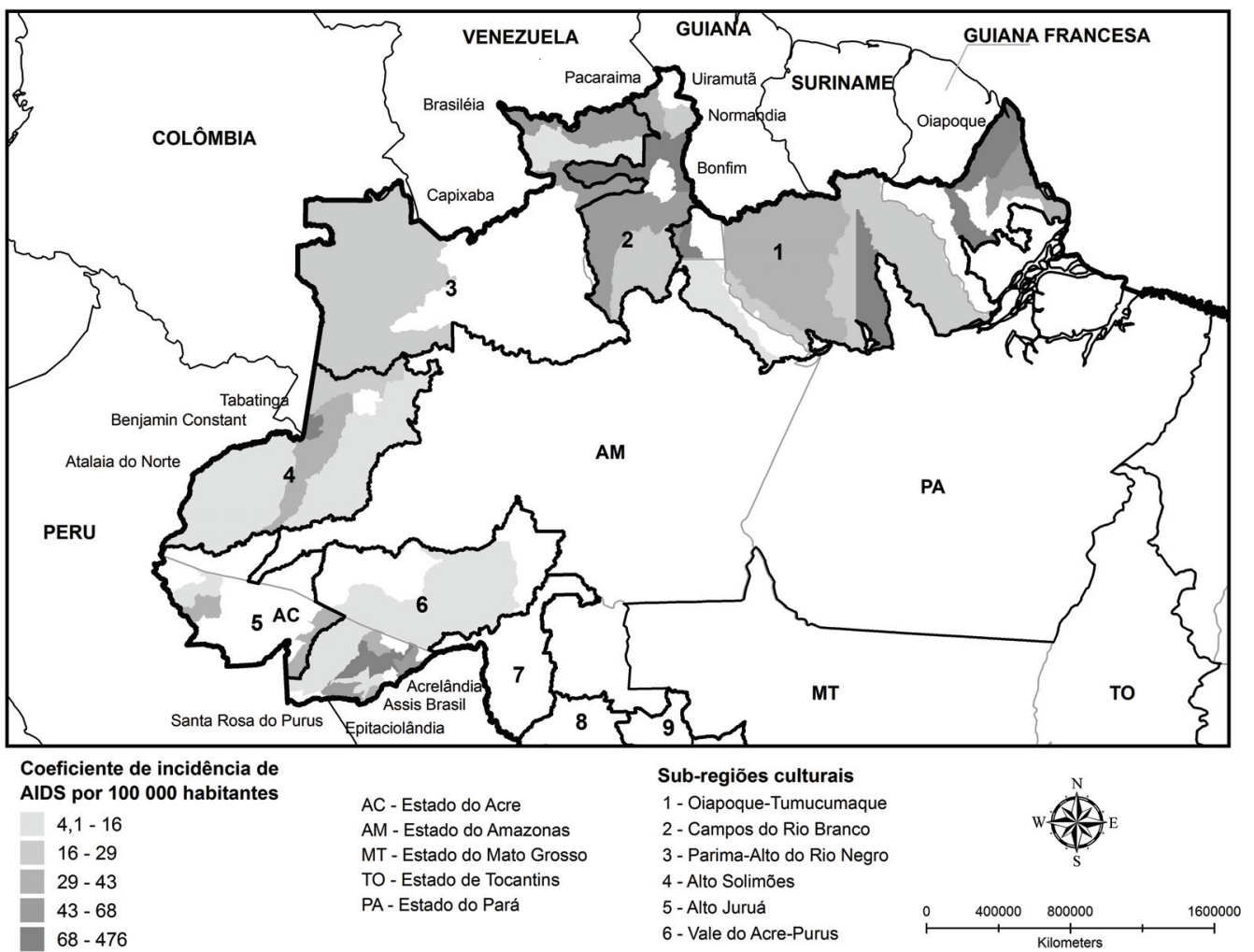

FIGURA 4. Distribuição espacial do coeficiente de incidência de AIDS na faixa de fronteira brasileira da área Centro-Oeste, Brasil, 1990 a 2003

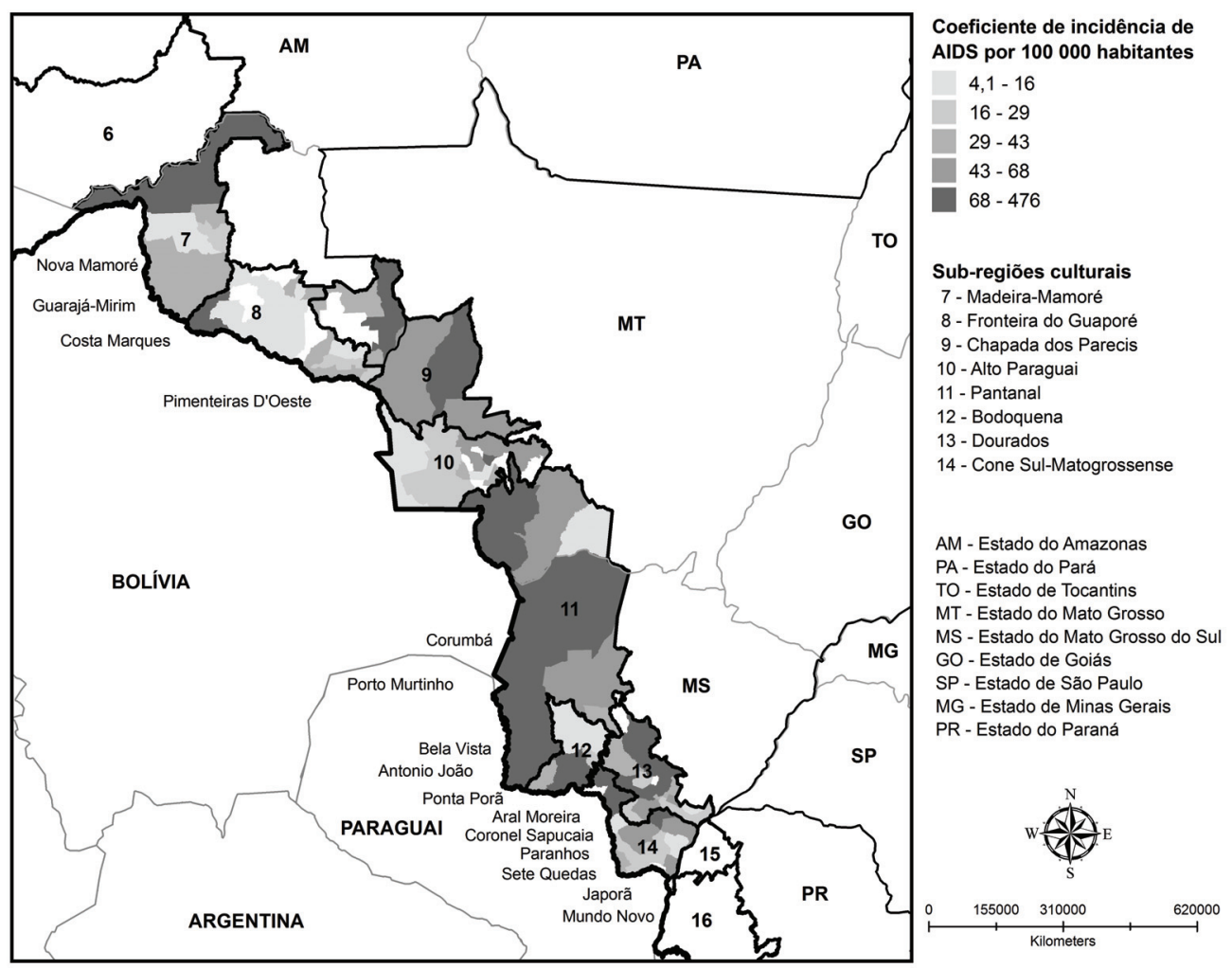


FIGURA 5. Distribuição espacial do coeficiente de incidência de AIDS na faixa de fronteira do Extremo Sul, Brasil, 1990 a 2003

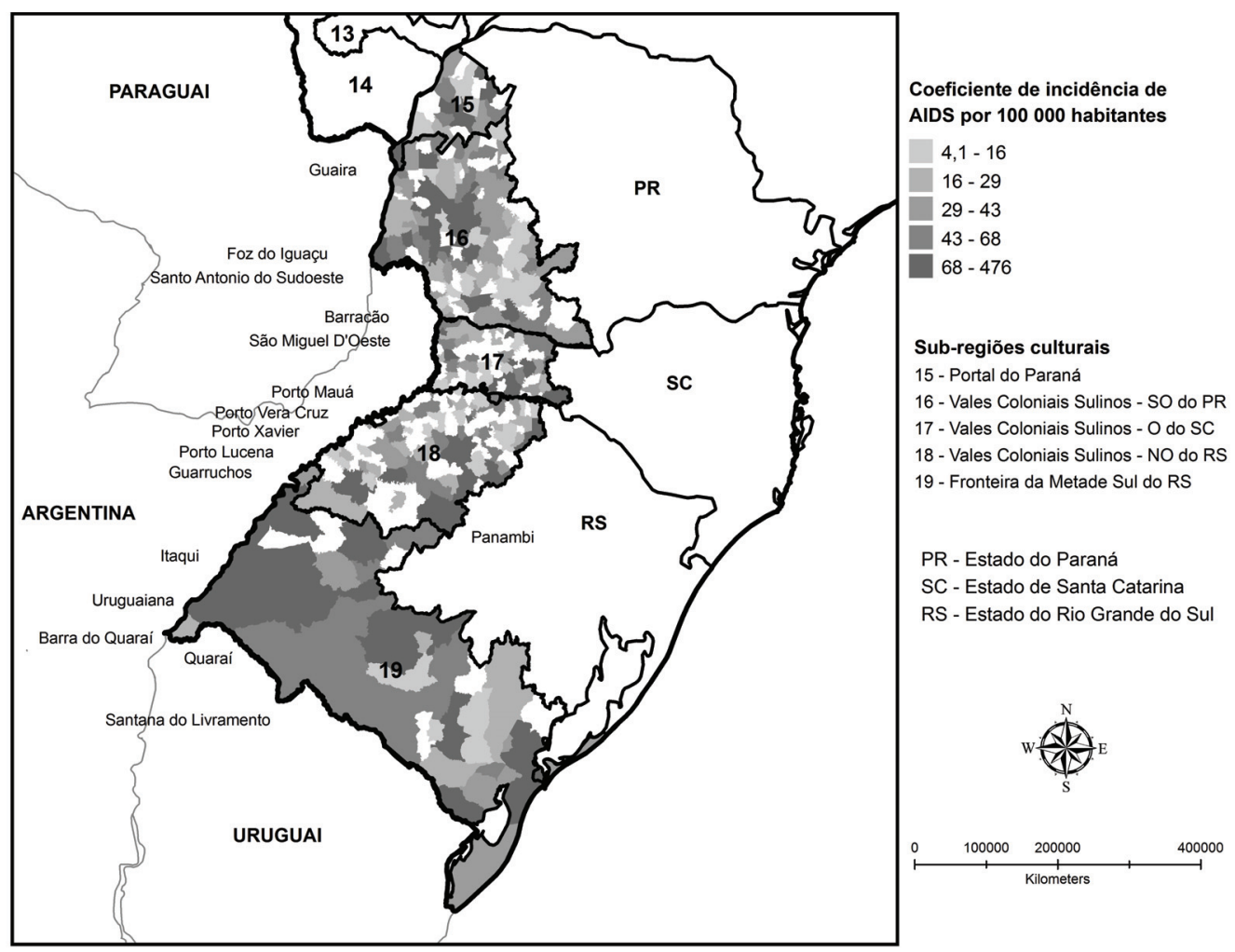

Guiana (17). Na tríplice fronteira PeruColômbia-Brasil (Estado do Amazonas), a falta de oportunidades sociais, o narcotráfico e a guerrilha (Frente Bolivariana de Libertação, Forças Armadas Revolucionárias da Colômbia e Exército Nacional de Libertação) provocam migrações para a Cidade de Tabatinga, para as cidades da região do Alto Solimões e para Manaus, onde a mortalidade proporcional por causas externas, especialmente por homicídios, é alta $(3,18)$. A fronteira com o Paraguai, por sua vez, é marcada pela degradação do ambiente provocada pela monocultura de soja, incluindo a soja transgênica, que provoca a migração de camponeses em busca de trabalho rural no lado brasileiro. Na tríplice fronteira Argentina-Paraguai-Brasil (Estado do Paraná), há relatos de atividade do crime organizado internacional, de comércio ilegal, de exploração sexual infantil e adolescente, de tráfico de seres humanos e de tráfico de drogas e de armas (4). Os resultados deste estudo mostraram que as sub-regiões com os maiores coeficientes de incidência de AIDS, em cada área, estão localizadas nas tríplices fronteiras do Brasil com outros países da América do Sul.
A paisagem da faixa de fronteira promove migrações de pessoas com poucas qualificações profissionais, que se evadem de espaços geográficos degradados em busca de melhores condições de vida ou de subsistência, e que se deslocam para municípios onde, supostamente, encontrarão novas trajetórias de vida ou novas oportunidades. Entretanto, dependendo do destino escolhido, os migrantes podem ter dificuldade para conseguir trabalho formal - oportunidade - e para ser incluídos na cadeia de produção econômica. Uma parcela da faixa de exclusão social é composta por pessoas que não conseguem trabalho formal e que não exercem atividades informais toleráveis pelo senso comum. Nesse sentido, é importante destacar que o fenômeno de "pauperização" do padrão de incidência da AIDS representa a presença de membros da faixa de exclusão social na cadeia de transmissão de AIDS no Brasil. É preciso identificar os municípios que atraem os fluxos migratórios, pois, além do valor simbólico no imaginário dos migrantes, eles apresentam grande conectividade na rede de produção de economia e na difusão de epidemias como a AIDS. Dentre os vários referenciais teóricos acerca da dinâmica humana sobre o território, merece destaque a teoria de localidades centrais, fundamentada no materialismo histórico, proposta por Walter Christaller, que se baseia na interação entre comunidades/ localidades e que aborda os determinantes dos fluxos motivados pelo consumo de bens e de serviços. Milton Santos, por sua vez, idealizou a teoria dos dois circuitos da economia, em que um circuito é constituído pelas localidades economicamente mais capazes - localidades centrais, que atuam como distribuidores regionais de bens e de serviços-e o outro circuito é formado pelo conjunto de localidades dependentes das facilidades das localidades centrais (19). Ambas as teorias contribuem para a construção do conhecimento estratégico necessário à diminuição das desigualdades regionais, à redução das vulnerabilidades sociais e ao enfrentamento da epidemia brasileira de AIDS na faixa de fronteira brasileira.

Dentre os vários programas e ações do Ministério da Saúde do Brasil, a Rede de Laboratórios de Fronteiras, o Sistema Integrado de Saúde das Fronteiras e os Distritos Sanitários Especiais Indígenas têm importância estratégica na assistên- 
cia às populações da faixa de fronteira. A Rede de Laboratórios de Fronteiras, composta atualmente por 12 laboratórios, representa um aperfeiçoamento dos serviços de diagnóstico de AIDS, por aumentar a capacidade de detecção de casos e a disponibilidade de exames laboratoriais de diagnóstico e por melhorar o sistema de informações de saúde. A distribuição geográfica desses laboratórios ainda é heterogênea, pois há quatro laboratórios na área Amazônica, três na área Centro-Oeste e cinco na área Extremo Sul; por um lado, os laboratórios localizados na área Amazônica cobrem grande extensão territorial, com várias reservas naturais e vários parques indígenas, além de atender municípios pouco povoados. Por outro lado, os laboratórios situados na área Extremo Sul têm maior demanda, dada a concentração populacional e a magnitude da incidência de novos casos da epidemia de AIDS. Com a criação do SIS Fronteiras, em julho de 2005, o Ministério da Saúde pretendeu fortalecer as suas ações na faixa de fronteira, com o objetivo de desenvolver a infra-estrutura dos serviços de saúde, de melhorar o acesso e a utilização dos serviços de atenção, de identificar fluxos migratórios, de analisar o impacto de ações e de programas em uma escala local, de aperfeiçoar os sistemas de informações e de participar dos pactos de gestão dos serviços de saúde, principalmente das atividades de vigilância em saúde (20). A implantação dos Distritos Sanitários Especiais Indígenas, como um sub-sistema do Sistema Único de Saúde, teve como objetivo o desenvolvimento de programas específicos junto às populações indígenas, com foco nas doenças sexualmente transmissíveis, principalmente HIV/AIDS. Esses programas devem aperfeiçoar as atividades de vigilância epidemiológica junto aos municípios da faixa de fronteira (20), já que, tradicio- nalmente, as comunidades indígenas transitam de um lado para outro das fronteiras, motivadas pelos laços familiares, pelas práticas culturais e pelas práticas espirituais, formando uma geografia cultural desvinculada das delimitações geopolíticas.

Para potencializar os resultados, é recomendável que as estratégias do $\mathrm{Mi}$ nistério da Saúde estejam articuladas com as de outros ministérios, em ações cooperativas, à semelhança do Protocolo de Intenções celebrado, em 2004, entre o Ministério da Saúde, o Ministério da Defesa e o UNAIDS. Através desse protocolo, foi firmado um acordo de cooperação para desenvolver as ações de prevenção e de controle de DST/AIDS, com vistas à melhoria da qualidade da assistência às populações situadas em locais de difícil acesso. Os "Navios da Esperança" prestam assistência hospitalar às populações ribeirinhas da Amazônia, e o Programa Calha Norte pretende aumentar a presença do poder público no território (21). Focalizando a reversão do quadro de desigualdade e de exclusão social, o Programa de Desenvolvimento da Faixa de Fronteira (13), da Secretaria de Programas Regionais do Ministério da Integração Nacional, destina-se à melhoria das estruturas físicas, sociais e econômicas de tais localidades, cujas diretrizes multissetoriais incluem a integração das ações de saúde, que pode ser articulada com o SIS Fronteiras.

O Programa de AIDS das Nações Unidas cita a estimativa de 620000 indivíduos infectados pelo HIV no Brasil em 2005, destacando a importância das categorias de transmissão sexual para o controle da epidemia. Na Argentina, estimou-se que a transmissão do HIV pelo sexo não seguro ocorreu em 4 de cada 5 novos casos diagnosticados, e que $5 \%$ das novas infecções de HIV pelo uso de drogas injetáveis ocorreram em Bue- nos Aires entre 2003 e 2005. A epidemia de AIDS no Uruguai concentrou-se na capital Montevidéu e nos distritos de Canelones, Maldonado e Rivera, que apresentaram mais de $75 \%$ dos novos casos de AIDS. Esses casos resultaram, principalmente, da transmissão do HIV pelo sexo não protegido. No Paraguai, a maioria dos casos de AIDS ocorreu na capital Assunção e em áreas fronteiriças com a Argentina e o Brasil, na tríplice fronteira, majoritariamente no sexo masculino. Na Bolívia, no Chile, na Colômbia, no Equador e no Peru a infecção pelo HIV ainda é prevalente entre homens que fazem sexo com outros homens. Estima-se que, em 2007, 1,6 milhão de pessoas estivessem vivendo com HIV, com 100000 novos adultos e crianças infectados pelo HIV e 58000 mortes pela AIDS na América do Sul (22).

A causalidade da transmissão do HIV e da AIDS, nas sub-regiões consideradas neste estudo, numa visão ecológica, é condicionada pelas características sociais, econômicas e culturais, além da infraestrutura e do acesso aos serviços de saúde das comunidades localizadas na faixa de fronteira brasileira. $\mathrm{O}$ aperfeiçoamento dos serviços de saúde pode ser uma estratégia de integração, considerando que a garantia constitucional de suporte à saúde das pessoas, mediante políticas públicas, revela a ação do Estado e a ocupação efetiva do território. $\mathrm{O}$ enfrentamento da epidemia de AIDS na faixa de fronteira brasileira é um tema complexo e sensível, porque requer a exposição de iniquidades, de violências e de práticas ilegais, principalmente nas áreas mais distantes do eixo políticoadministrativo do país.

Agradecimentos. Ao Programa Nacional de Doenças Sexualmente Transmissíveis e AIDS pelas informações sobre os casos de AIDS notificados ao Ministério da Saúde.

\section{REFERÊNCIAS}

1. Brasil, Ministério da Saúde. Portaria $1120 / 05$ de 6 de julho de 2005. Institui o Sistema de Saúde das Fronteiras - SIS Fronteiras. Disponível em: http://portal.saude.gov.br/ portal/arquivos/pdf/Port_1120-05.pdf. Acessado em 13 de março de 2008.

2. Becker BK. Amazônia. São Paulo: Editora Ática; 1990.
3. Oliveira MM. A mobilidade humana na tríplice fronteira: Peru, Brasil e Colômbia. Estud Av. 2006;20(57):183-96.

4. Sprandel MA. Brasileiros na fronteira com o Paraguai. Estud Av. 2006;20(57):137-56.

5. Brasil, Congresso Nacional. Lei 6634 de 2 de abril de 1979. Dispõe sobre a faixa de fronteira. Diário Oficial da União 1979. 3 de maio:6113. Disponível em: http://www.cna. org.br/site/noticia.php?n=1520. Acessado em outubro de 2008.

6. Peiter PC. A geografia da saúde na faixa de fronteira continental do Brasil na passagem do milênio [tese de doutorado]. Rio de Janeiro: Instituto de Geociências da Universidade Federal do Rio de Janeiro; 2005. Disponível em: http:/ / 
www.igeo.ufrj.br/gruporetis/pdf/tesebres paulo.pdf. Acessado em 13 de março de 2008.

7. Castro J. Geografia da fome: dilema brasileiro — pão ou aço. 10a ed. Rio de Janeiro: Antares Achiamé; 1980.

8. Rodrigues AL Jr, Castilho EA. A epidemia de AIDS no Brasil, 1991-2000: descrição espaçotemporal. Rev Soc Bras Med Trop. 2004;37(4): 312-7.

9. Arbex J Jr, Tognolli CJ. O século do crime. São Paulo: Jinkings Editores Associados; 1996

10. Parker R. Na contramão da AIDS: sexualidade, intervenção, política. São Paulo: Editora $34 ; 2000$.

11. Barcellos C, Peiter P, Rojas LI, Matida A. A geografia da AIDS nas fronteiras do Brasil. Campinas: Ministério da Saúde/CN DST/ AIDS; Population Council e USAID; 2001. Disponível em: http://www.igeo.ufrj.br/ gruporetis/gruporetis/modules/wfsection/ article.php?articleid=3. Acessado em 13 de março de 2008.

12. Chatfield C. The analysis of time series: an introduction. Florida: Chapman \& Hall/CRC; 2004.

13. Brasil, Ministério da Integração Nacional, Secretaria de Programas Regionais, Programa de Desenvolvimento da Faixa de Fronteira. Proposta de reestruturação do Programa de Desenvolvimento da Faixa de Fronteira. Brasília: Ministério da Integração Nacional;
2005. Disponível em: http:/ /www.integracao. gov.br/publicacoes/index.asp. Acessado em 13 de março de 2008.

14. Brasil, Ministério da Saúde, Conselho Nacional de Saúde. Resolução 196 de 10 de outubro de 1996. Prova as diretrizes e normas regulamentadoras de pesquisa envolvendo seres humanos. Diário Oficial da União 1996. 16 de outubro.

15. Libault A. Geocartografia. São Paulo: Companhia Editora Nacional; 1979.

16. Robinson AH, Morrison JL, Muehrcke PC, Kimerling AJ. Elements of cartography. New York: John Wiley \& Sons; 1995.

17. Rodrigues F. Migração transfronteiriça na Venezuela. Estud Av. 2006;20(57):197-207.

18. Rodrigues-Júnior AL. Geoepidemiologia da AIDS e das doenças oportunistas transmissíveis na faixa de fronteira brasileira [tese de livre-docência]. Ribeirão Preto: Universidade de São Paulo; 2007. Disponível em: http:// www.teses.usp.br/teses/disponiveis/livre docencia/17/tde-29112007-070303/. Acessado em 13 de março de 2008.

19. Costa WM. Geografia política ou geopolítica: discursos sobre o território e o poder. São Paulo: Editora HUCITEC; 1992.

20. Brasil, Ministério da Saúde, Secretaria de Vigilância em Saúde. Vigilância em saúde no SUS: fortalecendo a capacidade de resposta aos velhos e novos desafios. Brasília: Mi- nistério da Saúde; 2006. Disponível em: http:/ / www.google.com/url?sa=t\&source= web\&ct=res\&cd $=2 \&$ url $=$ http $\% 3 \mathrm{~A} \% 2 \mathrm{~F} \% 2 \mathrm{Fpo}$ rtal.saude.gov.br\%2Fportal\%2Farquivos $\% 2 \mathrm{~F}$ pdf\%2Flivro_nova_vigilancia_web.pdf\&ei=b 5sAScGhBYsef6hgOUN\&usg=AFQjCNH 5L bhXpf2P0I9HskmOjnHK9Ew1A\&sig2=heaPF7fck3MpLgXn1I76w . Acessado em 23 de outubro de 2008

21. Brasil, Ministério da Saúde, Programa Nacional de Doenças Sexualmente Transmissíveis e AIDS. Resposta +: experiências do Programa Brasileiro de AIDS. Brasília: Ministério da Saúde; 2006. Disponível em: http:/ / www.aids.gov.br/final/biblioteca/resposta/ resposta_2005.pdf. Acessado em 24 de outubro de 2007.

22. World Health Organization, Joint United Nations Programme on HIV/AIDS. AIDS epidemic: update 2007. Disponível em: http:// data.unaids.org/pub/EPISlides/2007/ 2007 epiupdate en.pdf. Acessado em 13 de março 2008.

Manuscrito recebido em 20 de dezembro de 2007. Aceito em versão revisada em 24 de março de 2008.

ABSTRACT Objective. To study the AIDS epidemic in Brazil's border areas, from the spatial and temporal perspective.

AIDS along Brazil's borders, 1990-2003

Key words
Methods. This was an ecological study in which the cases of AIDS reported to the Ministry of Health of Brazil from 1990-2003 were grouped according to "hunger areas" as defined by Josué de Castro in the 1940s and according to 19 cultural subregions. Spatial assessment was based on incidence rates for border municipalities; temporal assessment considered the absolute number of cases occurring quarterly from 1990-2003 in each of the hunger areas studied (Extreme South, Midwest, and Amazon).

Results. During the study period, 7973 cases of AIDS were reported from the Brazilian border areas: 648 in the Amazon area, 1579 in the Midwest, and 5746 in the Extreme South (populations of 668 098, 895 489, and 2769 361, respectively). The subregions with the highest AIDS incidence rates in each of the three border areas were those near triple-borders, between more than two Latin American countries. Sexual transmission was predominant, with heterosexual transmission being the most frequent, followed by transmission by male homosexuality. These two categories accounted for $87.2 \%$ of the cases reported. The estimates of the trend parameter in the temporal analysis were $0.53(P<0.0001), 0.83(P<0.0001)$, and $3.47(P<0.0001)$, respectively, for the Amazon, Midwest, and Extreme South areas.

Conclusion. The improvement of health care services along Brazil's borders may be a strategy for territorial integration and for dealing with the AIDS epidemic, as long as social, economic, and cultural differences are taken into account.

HIV, acquired immunodeficiency syndrome, border areas, ecological studies, Brazil. 